\title{
Research on Performance of Automotive Air Conditioning Filter
}

\author{
Shurong $\mathrm{Liu}^{1}$ \\ ${ }^{1}$ School of Civil Engineering and Architecture, Wuhan University of Technology, Wuhan, Hubei, 430070, China
}

\begin{abstract}
Explains the main sources and types of air pollutants in automobiles, and introduces methods to evaluate the effect of automobile air filters on the purification of the above-mentioned automobile air pollutants, and the purification effects of existing automobile air filter products on the market Evaluate and discuss its purification level to provide a theoretical basis for the future formulation of relevant specifications.
\end{abstract}

\section{Introduction}

As one of the most important means of transportation in modern society, cars are closely related to people's daily life. People spend about $7 \%$ of their time in transportation. At the same time, with the popularization of environmental protection concepts, more and more Many people realize the importance of reducing resource consumption and sustainable development, which has also enabled new energy vehicles to develop vigorously around the world. With the gradual deepening of research on indoor air quality and the rapid growth of car ownership, people are paying more and more attention to the air quality in the car compartment. Like the built environment, HVAC systems play an important role in car thermal comfort and air quality; in modern vehicles, including new energy vehicles, the HVAC system is manually managed by passengers or automatically managed by the on-board control system. According to the external conditions (temperature, humidity, pollution), the air supply volume and the ratio of circulating air to fresh air can be adjusted.

Ordinary cars use the waste heat generated by the engine to heat the medium in winter to heat the air. Because there is no waste heat generated by the engine in the winter, the main heating method of new energy vehicles is to use the electric heating PTC installed inside the air conditioning assembly or use the heat pump air conditioner Heating and recirculating the gas in the car. However, in addition to heating, another major function of the air-conditioning system is to purify the air. Modern cars mainly filter and purify the air by car air filters. The installation of car air-conditioning filters is the most mainstream means of purifying the air in the car., The quality and filtering effect of the air-conditioning filter have become a major factor affecting the health of people in the car; how to ensure the thermal comfort of people in the car while also making the air in the car meet the requirements of human health has become one of the mainstream topics now .

Therefore, analyze the types and sources of air pollutants contained in the car, be familiar with the principles of air-conditioning filters, and whether the purification effect of the existing car air-conditioning filters can meet the health requirements of people in the car according to relevant standards It is very necessary to develop relevant evaluation methods.

\section{Sources and types of main pollutants in automobile cabins}

There are many types of pollutants in automobiles, and their sources are also different. The air pollutants in the car mainly come from the following three aspects: one is the pollutants released in the interior parts and parts materials of the car; the second is the pollutants in the atmospheric environment entering the car; the third is the car itself during use The generated pollutants enter the car [1].

(1) Pollutants released from automotive interior parts and parts materials. Automotive interior materials mainly include: composite materials, leather, rubber materials, fabrics, adhesives, and thermal insulation materials. The organic volatile components contained in these materials are gradually released into the car, resulting in an increase in the concentration of pollutants in the car. These volatile organic pollutants mainly include benzene, toluene, xylene, formaldehyde, acetone, aromatic hydrocarbons, etc., which are also the main source of unpleasant odors in cars, especially in new cars. In summer, the temperature outside and inside the car is relatively high. In the case of sun exposure, it will accelerate the volatilization of formaldehyde and benzene series, which will increase the degree of pollution in the car and reduce the comfort of people in the car. After testing and analysis, there are dozens of volatile organic compounds (VOC) contained in automotive paint. Being in such an environment for a long time not only affects the comfort of passengers, but also sometimes causes headaches, fatigue and other symptoms. In severe cases, it can cause major diseases such as leukemia and harm human health.

(2) Pollutants in the atmospheric environment enter the car. The internal environment of the car is greatly affected by the background environment outside the car. If the seal is not tight or the window is opened, the pollutants in the

* Corresponding author: 2100270351@qq.com 
atmospheric environment will enter the car with the airflow, causing pollution to the air inside the car. The pollutants in the atmospheric environment mainly include suspended particulate matter produced by dust, organic matter, nitrogen oxides, carbon oxides, and sulfides emitted by various factories. In the case of haze weather or poor outdoor air quality, when the vehicle is driving, when the window is opened or the air conditioning system is turned on, the pollutants from outside the vehicle will enter the vehicle with the airflow, thereby causing pollution to the air inside the vehicle. Studies have shown that due to the existence of infiltration, unpurified external air will directly enter the interior of the cabin. The main infiltration location is near the front pedals, and as the speed increases, the amount of air that penetrates into the car will also It rises accordingly.

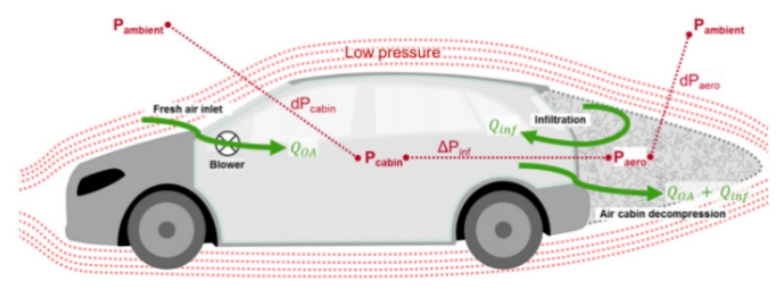

Fig. 1. Cycle diagram

(3) The pollutants emitted by the car itself enter the car during its use. During the operation of a car, gasoline combustion will emit a large amount of exhaust gas. The exhaust gas mainly contains carbon oxides, nitrogen oxides, particulate matter, benzene series, aromatic hydrocarbons, etc. Studies have shown that automobile exhaust can pass through the rear of the car. The gap enters the interior of the car [2].

\section{Research status of automobile air- conditioning filter}

Different from ordinary non-woven air filters used for engine air filters, car air-conditioning filters inject static electricity on the non-woven fibers so that the filter fibers are microscopically charged and the whole is electrically neutral. The fine particles themselves also have a small amount of charge, and the fiber of the filter can absorb the tiny particles in the air to achieve the effect of purifying the air[3].

Since its first application in Mercedes-Benz R-129 models in 1988, automotive air-conditioning filters have developed rapidly [4]. The air filter used in the passenger compartment can effectively block dust, pollen and pollutants from entering the car compartment, while ensuring the health of the people in the car, but also effectively protecting the car air conditioning system[5]. Up to now, as people's requirements for cabin air quality increase, automotive air conditioning filters have been constantly developing and innovating.

In order to regulate the performance and use of such products, the German Society of Engineers has successively formulated the industrial standard DIN71460-1/2; and the previous version has been highly recognized in the market and has been applied in many aspects. At present, the domestic automotive air conditioning filter testing standards are mainly based on the $\mathrm{GB} / \mathrm{T}$ 32085.1-2015 and GB/T 32085.1-2015 formulated by the China Automotive Research Institute. Such standards basically adopt the international standard ISO/TS 11155-1: 2001. Detection methods. These two standards respectively describe the detailed detection methods for the dust filtration test and the gas filtration test.

\section{Introduction to Test Experiment System}

\subsection{Overview of the test system}

In order to correctly evaluate the removal effect of automotive air conditioning filters on particulate matter and gaseous pollutants, with reference to the international standard ISO 11155-1-2009, a test bench for testing the performance of automotive air conditioning filters was built.
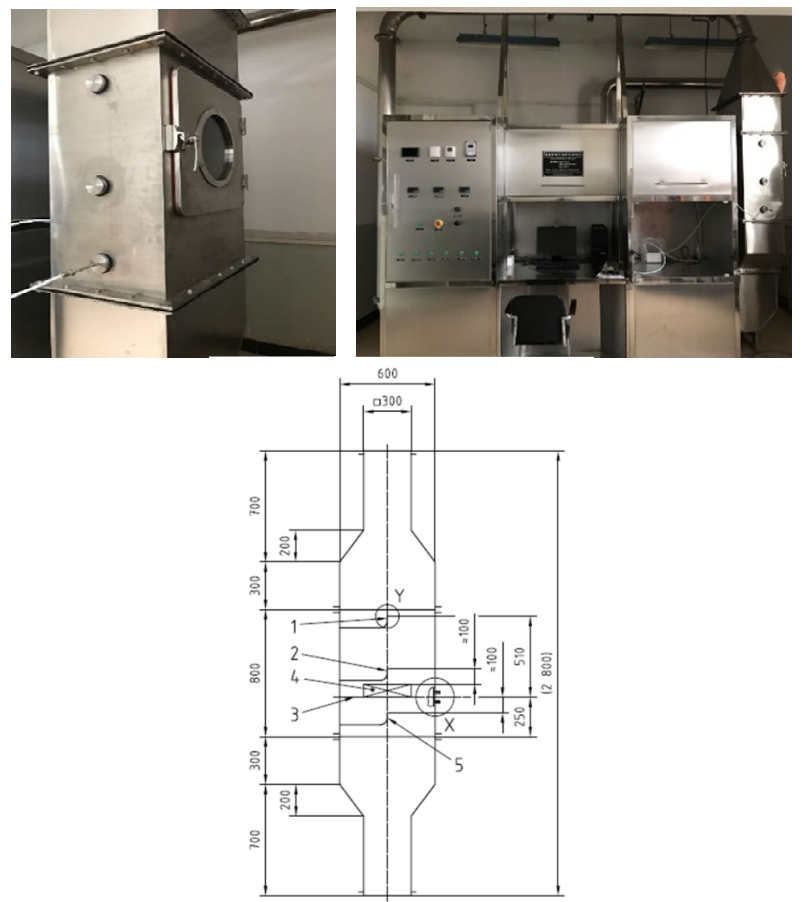

Fig. 2. Equipment diagram

1. Test dust injection port $X$ filter resistance test tube

2. Upstream sampling probe (connected to counter or gas analyzer)

3. Test the filter mounting plate $\mathrm{Y}$ for dust injection and mark the gas nozzle

4. Test the filter

5. Downstream sampling probe (connected to counter or gas analyzer)

The main body of the road vehicle filter test system consists of a pre-filter system (atmospheric particulate matter filtration/atmospheric chemical pollutant system), particulate matter/test gas pollutant generation system, generating tube, upstream detection port, static pressure ring, and road vehicle filter to be tested. It consists of 
cleaner, downstream detection port, centrifugal fan and exhaust gas processing unit. The experimental pipeline is made of mirror stainless steel to ensure the corrosion resistance of the pipeline. At the same time, the test section has a transparent window design to ensure aesthetics and can also be used as an observation window to monitor the internal conditions of the pipeline during the test.

\subsection{Pollutant generating device}

The main difference between the above two types of test systems is the pollutant generating device, which will be introduced separately below.

\subsubsection{Particulate matter generating device}

\section{(1) Dust from solid particles}

A2 solid dust adopts PALAS company's BEG1000 new automatic dust generator (see technical manual for details), a nozzle dust generator specially designed for A2 solid particles for automotive filter testing, which transports the solid dust to the inlet through the dust feeder, At the feed inlet, the nozzle accelerates to form a high-speed airflow, using the principle of siphon and the shearing force of the airflow, the dust is fully dispersed and input into the particulate matter test pipeline. The dust generator can be based on the dust holding capacity of the road car air-conditioning filter. Test requirements, adjust the dust feeding rate, can realize efficiency test and dust holding test.

In order to solve the problem of agglomeration and compaction during the dust loading process of the dust feeder, a special dehumidification and dust loading workbench is designed to achieve a relative humidity of less than $30 \%$, which meets the needs of operation and storage functions.

(2) Dust from liquid particles

DEHS adopts the automatic control PALAS liquid particulate dust generator PLG2000, a constant pressure and constant flow Collison nozzle atomizer, the compressed air is substituted into the nozzle through the annular gap, and the shear force between the air and the liquid of the Collison nozzle changes the liquid state. The particles are dispersed to form fine aerosol particles. It is suitable for liquid dust such as DEHS, DOP, etc. The performance parameters can refer to the technical manual.

\subsubsection{Gaseous pollutant generating devices}

(1) Peristaltic pump type constant pressure and constant flow generator

The organic pollutant generator is designed with the idea of heating and evaporating quantitative liquid pollutants (heat generation method). The main objects are organic pollutants that have no direct gaseous pollutants or gaseous pollutants that are unstable at room temperature and behave as pure liquids at room temperature. The design diagram is shown below, the pure liquid is injected into the evaporation chamber through the power supply device (peristaltic pump), the temperature of the evaporation chamber is adjusted by the PID controller to promote the organic liquid to evaporate into the gaseous state, and clean air is used as the carrier gas to pollute the gaseous state. Bring objects into the test system. Among them, the evaporation chamber needs to have the characteristics of anti-corrosion and high temperature resistance, and it is generally made of stainless steel.

The concentration and effect of pollutants in the peristaltic pump generator are mainly affected by three parameters: the pumping speed (rotation speed of the peristaltic pump), the temperature of the evaporation chamber and the flow rate of the carrier gas. In order to facilitate the control and simplify the operation, the temperature of the evaporation chamber and the flow of the carrier gas are usually kept constant, and the pumping speed is adjusted to meet the requirements of different concentrations. In actual use, the set temperature of the evaporation chamber is higher than the contaminant liquefaction temperature, which is monitored by a highprecision thermocouple and PID controller. At the same time, the gas flow is controlled by a high-precision mass flow controller (MFC) to ensure the stability of the gas flow.

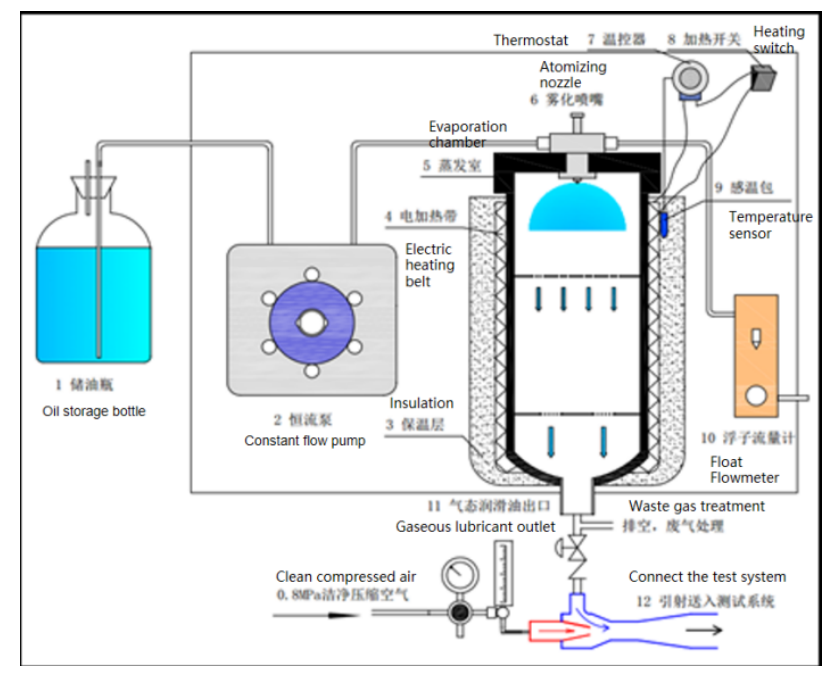

Fig. 3. Generator schematic

(2) Standard gas cylinder gas pollutant generator

For chemical pollutants that are gaseous at room temperature (such as SO2, H2S, NH3, etc.), a compressed gas cylinder containing a single test gas with a specific concentration can be used directly. The mass flow controller is used to control the specific concentration of gas in the cylinder to obtain the target loading concentration. At the same time, for the consideration of experimental safety and personnel health, the use of highpurity gas requires strict attention. All connecting valves, pipe fittings and regulators should be made of corrosionresistant materials. Try to choose the same type of stainless steel (above 316) from the same manufacturer Pipe fittings to avoid Electrochemical corrosion of the pipeline. In addition, for individual gaseous organics, it is recommended to contact local suppliers to determine whether they can provide a single VOC test gas at the required concentration level. In this case, no dilution is required and the gas generation process will be simplified. 


\subsection{Testing software}

Since the test process and test report of the road vehicle filter are basically fixed, in order to reduce the difficulty and workload of the test, a software for road vehicle filter testing is provided, and the operation on the software is used to achieve automatic Testing, data processing and completion of test reports. The expected operation interface of the software is as follows:

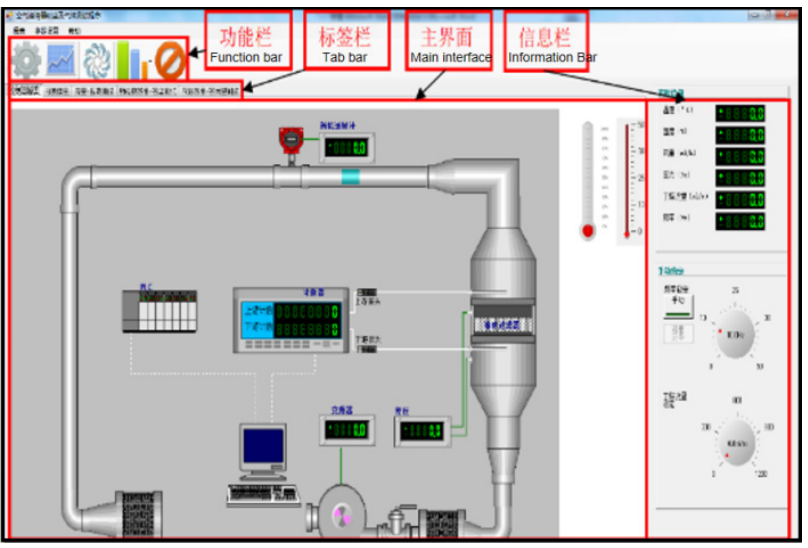

Fig. 4. Operation interface

\section{Test results and efficiency analysis of some automotive filters on the market}

\subsection{Particulate matter (A2 dust) filtration efficiency}

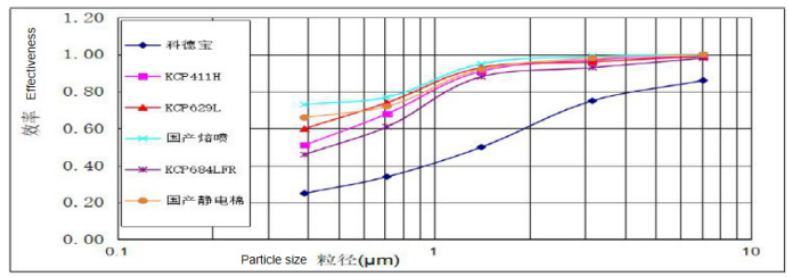

Fig. 5. Filtration and classification efficiency of particulate matter of different products

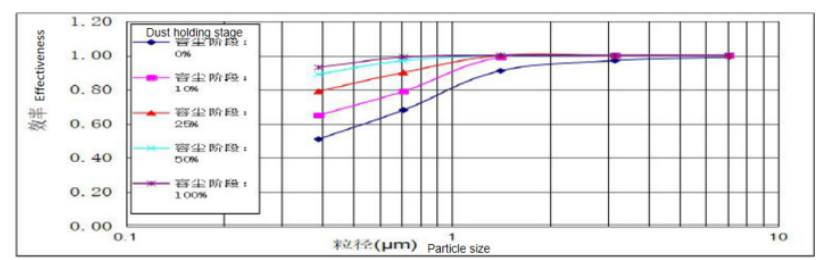

Fig. 6. Filter and classification efficiency of particulate matter in different dust holding stages of a product

\subsection{Gaseous pollutants (butane) filtration efficiency}

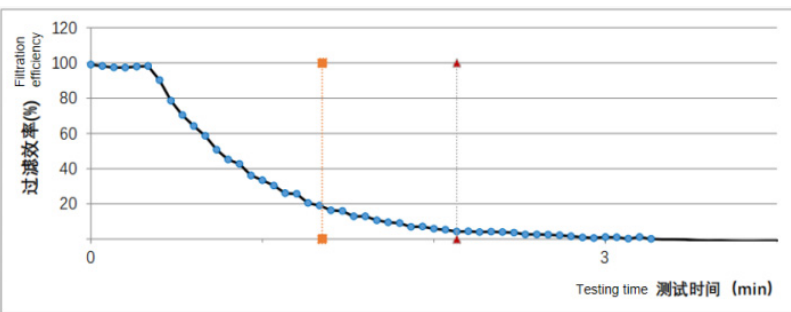

Fig. 7. Filter efficiency of gaseous pollutants of a product

\subsection{Comparison and analysis of results}

From the particulate matter purification efficiency diagram of each product, it can be seen that the particulate matter filtration efficiency of different products is different. The test simulation test temperature is $23^{\circ} \mathrm{C}$, humidity $50 \%$, the test uses A2 dust, and it is dried at $105^{\circ} \mathrm{C}$ for 4 hours. The filter efficiency of each product particulate matter in the actual air-conditioning operation process can be approximated by this graph. With the increase of the initial dust holding capacity (dust holding stage) of the filter, the filtration efficiency of each particle size will increase. This is because as the dust holding capacity increases, that is, the number of particles deposited on the surface of the fiber increases, the absorption The surface area will also increase accordingly, and the filtration efficiency will increase accordingly, but due to the obstruction of dust accumulation, the resistance of the filter will also increase. Therefore, in the actual use process, as the use time increases, the filtration efficiency of the automobile air-conditioning filter will gradually increase. However, in order to ensure the normal operation of the automobile air-conditioning filter, it is also necessary to replace the new filter material regularly to ensure Moderate resistance.

In contrast, it can be seen from the figure that the filtering efficiency of gaseous pollutants for automobile air-conditioning filters decreases as the use time increases, and the efficiency decays quickly. This is mainly because the filter mainly relies on activated carbon. The adsorption effect filters gaseous pollutants. As the absorption time is extended, the fine pores on the surface of the activated sharp decrease in filtration efficiency, which makes the replacement of automobile air-conditioning filters more frequent.

\subsection{Prospects of relevant standards for automotive air conditioning filters}

Although there are currently international standards for grading the efficiency of building air-conditioning filters, there is no relevant efficiency standard for automotive airconditioning filters. As people pay more and more attention to the length of the car's use time and the environmental air quality in the cabin, this Research believes that it is also necessary to formulate relevant vehicle air-conditioning filter efficiency standards. 


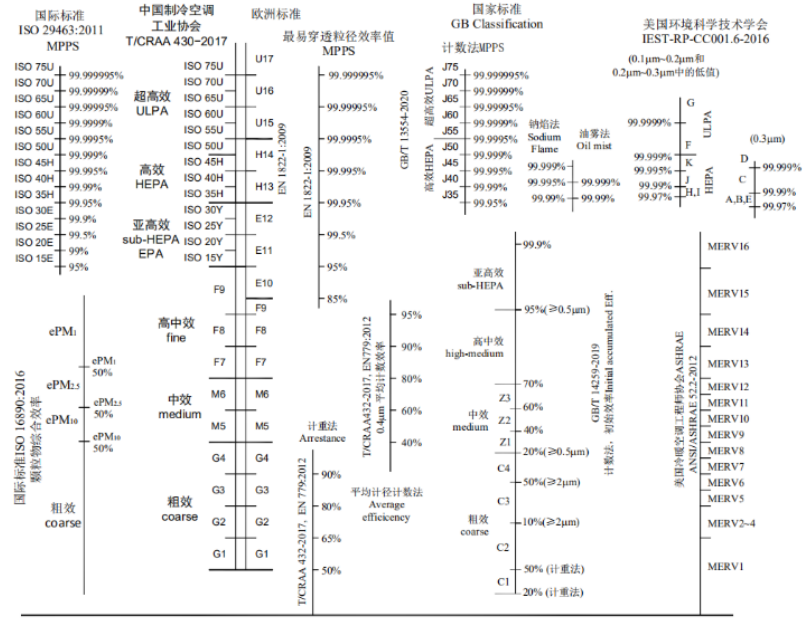

Fig. 8. Efficiency classification standards for building airconditioning filters

According to the test results, it can be seen that the filtration efficiency of most products in the new delivery state (dust holding stage $0 \%$ ) for each particle size is above $60 \%$, and because the experiment uses the MPPS most easily penetrated particle size efficiency value, Therefore, the straight line (red part) of the car air-conditioning filter can be made in the above figure.

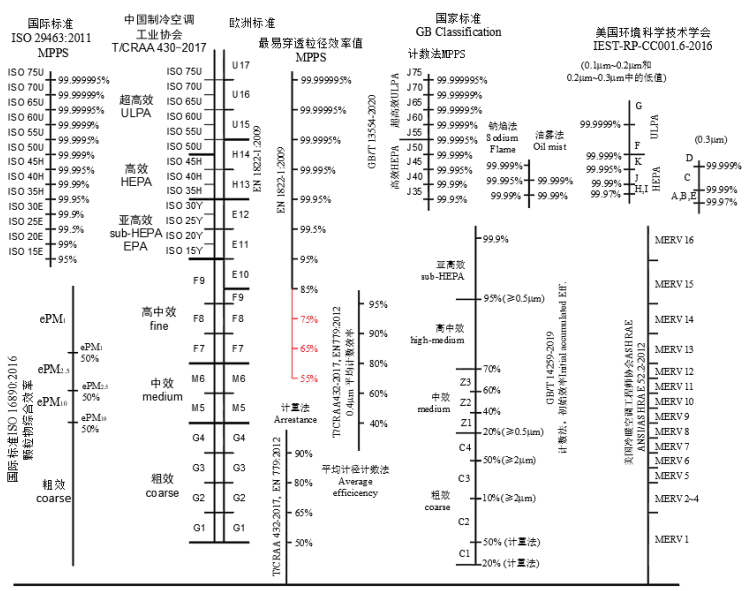

Fig. 9.Illustration of car air conditioner(red part)

\section{Conclusion}

It can be seen that the filtration efficiency of automotive air-conditioning filters for particulate matter is within the efficiency range of high- and medium-efficiency filters, that is, automotive air-conditioning filters that meet this range can be regarded as qualified products, and related automotive air-conditioning filters are formulated based on this The device standard is to standardize the manufacturer's requirements for the manufacture of such products.

\section{References}

1. Qian Jiayan, Wu Yiyu, Jiang Zengjie, et al. Research progress on sources and control measures of pollutants in vehicles[J]. Clean the World, 2020, 35(12): 105-107.
2. <ATMENV-D-20-02295_reviewer.pdf $>[\mathrm{J}]$.

3. Jiang Mingxiu. Research on the filtration performance of automobile air-conditioning filters under different heat and humidity environments [D]. Tianjin University, 2009.

4. Wen Wenyong. Research on the performance test method of automobile air-conditioning filter[D]. Tianjin University, 2008.v

5. Qian Cheng. Automobile air-conditioning filter and its latest development[J]. Industrial Textiles, 2009, 27(03): 1-4. 